\title{
Design of Miniaturized Band-Pass Filter with Composite Right/Left-Handed Transmission Line
}

\author{
Meng Zhang, Fang Zhang, Xingfang Luo \\ Laboratory of Nanomaterials and Sensors, School of Physics, Communication and Electronics, Jiangxi Normal \\ University, Nanchang, China \\ Email: xfluo@jxnu.edu.cn
}

Received January 2015

Copyright @ 2015 by authors and Scientific Research Publishing Inc.

This work is licensed under the Creative Commons Attribution International License (CC BY). http://creativecommons.org/licenses/by/4.0/

c) (i) Open Access

\begin{abstract}
In this paper, a new miniaturized wide-pass-band filter at $U$ band based on composite right/lefthanded transmission line (CRLH TL) is implemented. The CRLH TL contributed to a broadband filter is a balanced structure with a small size of only $1.428 \mathrm{~mm}^{*} 0.5530 \mathrm{~mm}$. The frequency characteristics are simulated and the results show that the $3 \mathrm{~dB}$ band width of the proposed filter is about 13.5 GHz from 45.8 GHz to $59.3 \mathrm{GHz}$. The insertion loss is quite flat through the pass-band and the return loss is relatively low.
\end{abstract}

\section{Keywords}

Miniaturized, Composite Right/Left-Handed Transmission Line, Bandpass Filter, Metamaterial

\section{Introduction}

Band-pass filters (BPF) are essential components in the modern radar and wireless communication system whose performance can greatly influence the behavior of the whole system. Therefore, small size, flat group delay, better frequency selection characteristic, and broadband filter are needed in practical Engineering. The resonator of the filter is the key device that determines the main characteristics of the filter.

In 2003, a resonator based on composite right/left-handed transmission line (CRLH TL) [1] is proposed by Caloz [2] [3], having attracted great interest from the scientific and engineering field owing to its potential applications of negative effective permeability. As is known, CRLH TL metamaterials are composed of lefthanded (LH) and right-handed ( $\mathrm{RH})$ components [4] [5], which has been approved to be able to realize miniaturization of structure [6] [7] utilizing its dispersive phase properties [8]. There are various researches on the ap- 
plication of the ultrawideband (UWB) band pass filter (BPF) with CRLH TL [9] [10]. The resonant mode of traditional TL (which is called right-handed TL.) resonator only can be positive and the resonant length is closely related to the size of the resonator. While, the resonant mode of CRLH TL can be any arbitrary integer, and the resonance frequency of CRLH TL only depends on the values of $L$ and $C$ in resonant unit, which results in significantly reducing the size of passive components. The CRLH TL has wide application prospect in the design of filter due to its characteristics of frequency selective and its balanced structure, and which opens up a new way for the design of broadband filter. Moreover, compared with the traditional TL resonator, the structure with CRLH TL has the advantage of low loss.

At present, millimeter band offers the benefits of the higher guaranteed data rates to some wireless technology, longer distance transmissions and more cost effectiveness than optic systems. With the development of ultra-high speed and high-capacity point-to-point communication systems in recent years, the research of millimeter wave communication is needed to satisfy the demand of broad market. In this paper, a microwave band-pass filter based on CRLH TL at U band is presented.

\section{Analysis and Design of the Proposed Bandpass Filter}

The CRLH TL in [2] consists of interdigital capacitance and grounding via stub, and the interdigital capacitor is a multi-finger periodic structure as shown in Figure 1.

An approximate expression for the interdigital capacitance $C_{L}$ is given by [11]

$$
\begin{aligned}
& C_{L}=\left(\varepsilon_{r}+1\right) L\left[(N-3) A_{1}+A_{2}\right], \\
& A_{1}=4.409 \tanh \left[0.55(h / m)^{0.45}\right] * 10^{-6}(p F / \mu m), \\
& A_{2}=9.92 \tanh \left[0.52(h / m)^{0.5}\right] * 10^{-6}(p F / \mu m) .
\end{aligned}
$$

where $N$ is the number of the interdigital. The proposed CRLH TL is fabricated on a microstrip substrate with dielectric constant $\varepsilon_{r}$ and thickness $h$.

In this paper, the obtained structure of the bandpass filter with CRLH TL is shown as Figure 2. A substrate with a relative dielectric constant $\varepsilon_{r}$ of 5.9 and a thickness $h$ of $0.1 \mathrm{~mm}$ is chosen for the filter design.

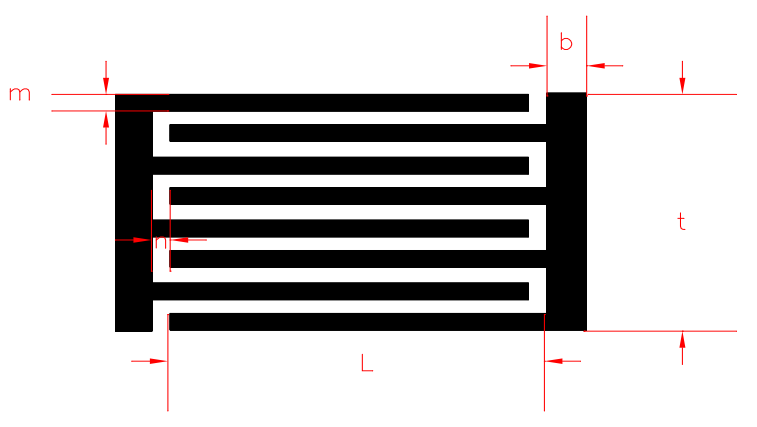

Figure 1. An interdigital capacitor configuration.

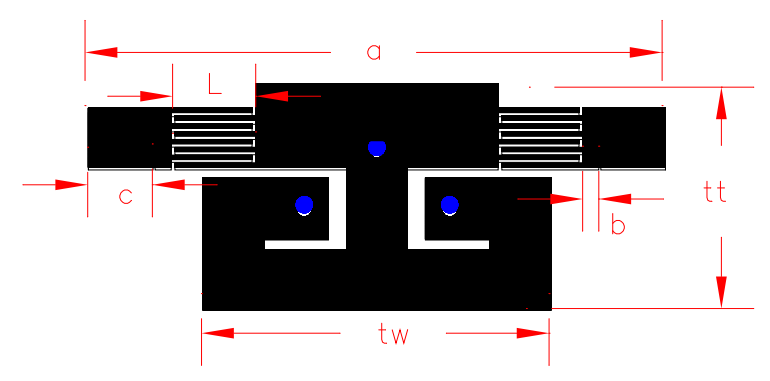

Figure 2. Layout of the proposed band-pass filter $(\mathrm{a}=$ $1.428 \mathrm{~mm}, \mathrm{~b}=0.04 \mathrm{~mm}, \mathrm{c}=0.16 \mathrm{~mm}, \mathrm{~L}=0.2 \mathrm{~mm}, \mathrm{tt}=$ $0.5530 \mathrm{~mm}$, tw $=0.86 \mathrm{~mm}$ ). 
The size of proposed filter is only $1.428 \mathrm{~mm} * 0.5530 \mathrm{~mm}$, which is smaller than the traditional filter, then the compact size has been achieved for low-cost microwave monolithic integrated circuit (MMIC) [12]. Figure 3 presents the modified T-typed equivalent circuit of a third-order inline coupling band-pass filter, which consists of one shunt resonator and two series resonators. It is seen here that the series capacitors for $C_{L}$ is realized by two identical interdigital capacitors, while the shunt inductor for $L_{L}$ is realized by a short-circuited stub. The resonant frequency of shut and series are $\omega_{s e}$ and $\omega_{\text {sh }}$ as

$$
\omega_{s e}=\frac{1}{\sqrt{L_{L} C_{R}}}, \quad \omega_{s h}=\frac{1}{\sqrt{L_{R} C_{L}}} .
$$

The propagation constant $\beta$ of the CRLH TL is given by [13]

$$
\beta=s(\omega) \sqrt{\omega^{2} L_{R} C_{R}+\frac{1}{\omega^{2} L_{L} C_{L}}-\left(\frac{L_{R}}{L_{L}}+\frac{C_{R}}{C_{L}}\right)},
$$

where $s(\omega)=\left\{\begin{array}{l}-1, \omega<\omega_{\tau 1}=\min \left(\omega_{s e}, \omega_{s h}\right) \\ +1, \omega>\omega_{\tau 2}=\max \left(\omega_{s e}, \omega_{s h}\right)\end{array}\right\}$.

It means that the CRLH TL based on the basic LC circuit supports a forward wave in the RH effect area $(\beta>0)$, where the structure works in the low-pass band, while a backward wave is in the LH effect area $(\beta<0)$, where the structure operates on the high-pass band. In general, there is a stop-band occurs between transition from LH to RH propagation. However, when $\omega_{s e}=\omega_{s h}$, the proposed CRLH TL filter in this paper is a balanced structure which has a transition frequency $\omega_{0}$ without stop-band.

The dispersion diagram of the obtained balanced CRLH TL filter is shown in Figure 4. The bandwidth of balanced CRLH TL is from high-pass left-handed cut-off frequency $\omega_{L}$ to low-pass right-handed cut-off frequency $\omega_{R}$ with no obvious stop-band, and a broad BPF is able to obtained. The cut-off frequencies ( $\omega_{L}$ and $\omega_{R}$ ) are given below,

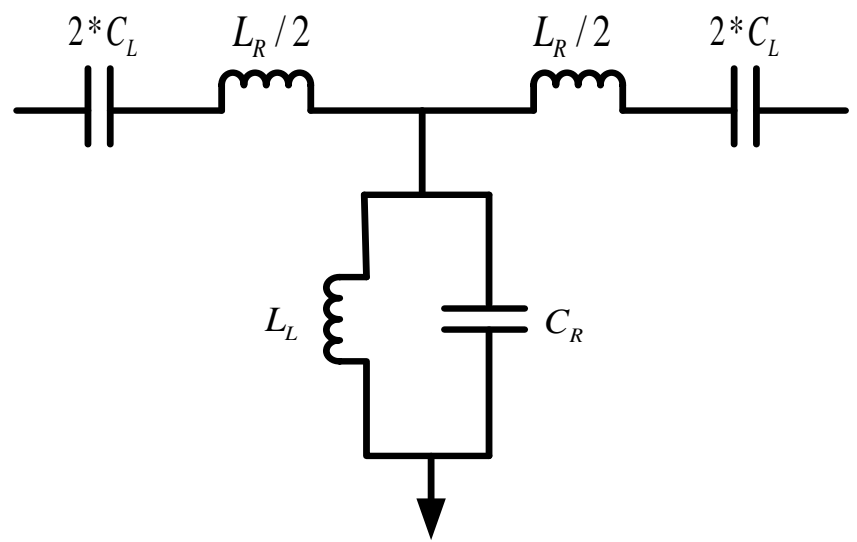

Figure 3. T-type equivalent circuit of the 3rd order band-pass filter.

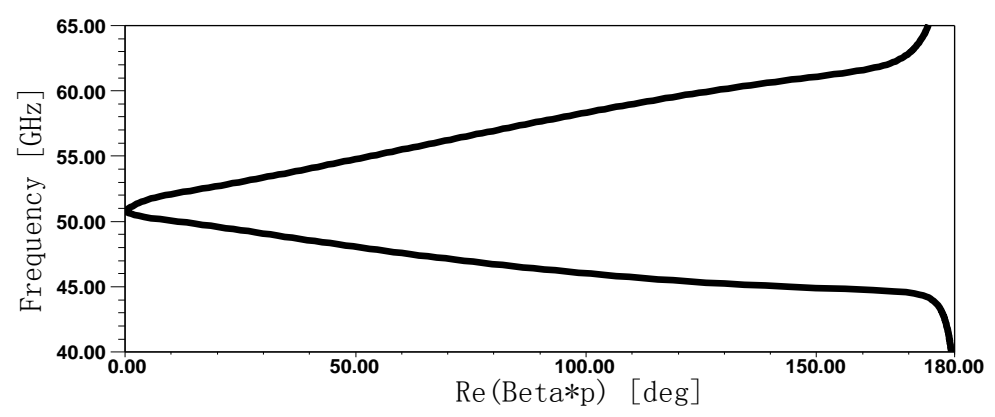

Figure 4. Dispersion diagram of composite right/left-handed transmission line. 


$$
\omega_{L}=\frac{1}{\sqrt{L_{L} C_{L}}}, \quad \omega_{R}=\frac{1}{\sqrt{L_{R} C_{R}}} .
$$

According to Equations (1)-(4), the finger width $m$, length $l$, and space $n$, as well as the number of the fingers $\mathrm{N}$ determines the dimensions of the interdigital capacitors, and the dimensions of the short-circuited stub, namely, the width and length, which results in an increase in the bandwidth.

\section{Results}

Figure 5 presents the simulated Scattering (S) parameter frequency responses to band-pass filter. It is observed that the proposed structure has a pass-band characteristic with two obvious transmission poles at $48.6 \mathrm{GHz}$ and 54.4 GHz which provides a good return loss. The $3 \mathrm{~dB}$ bandwidth of the implemented filter is about $13.5 \mathrm{GHz}$ from $45.8 \mathrm{GHz}$ to $59.3 \mathrm{GHz}$, and the relative bandwidth is about $25.7 \%$. Furthermore, the return loss is lower than $-13 \mathrm{~dB}$ and the insertion loss is relatively flat, implying a good matching impedance. The curve of variation of group delay over the pass-band is shown in Figure 6. Apparently, it is not a good practice to realize highly selective filters with proposed filter, which meets the broadband requirements.

\section{Conclusion}

A new $U$ band band-pass filter based on CRLH TL is presented in this paper. The proposed structure has a smaller size compared with traditional filter. A wide pass-band filter is obtained by using balanced CRLH TL structure, and the simulation results show that the proposed filter has a quite smooth group delay, and the insertion loss is relatively flat in the pass-band which shows that the ripple of pass-band is small enough, implying that the impedance matches better. The miniaturized wide band-pass filter using CRLH TL has certain reference value in the modern radar and wireless communication system of millimeter wave.

\section{Acknowledgements}

This work is supported by National Natural Science Foundation of China (Grant Nos. 51361013), Natural

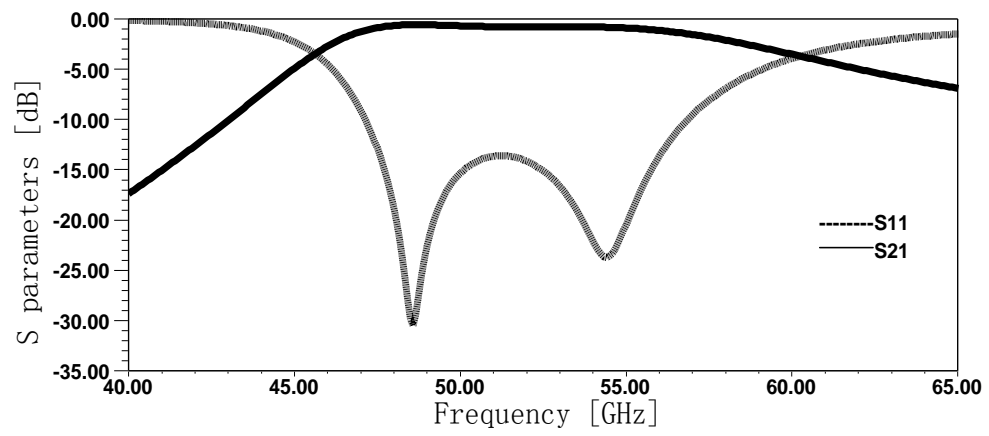

Figure 5. Scattering parameter of proposed band-pass filter.

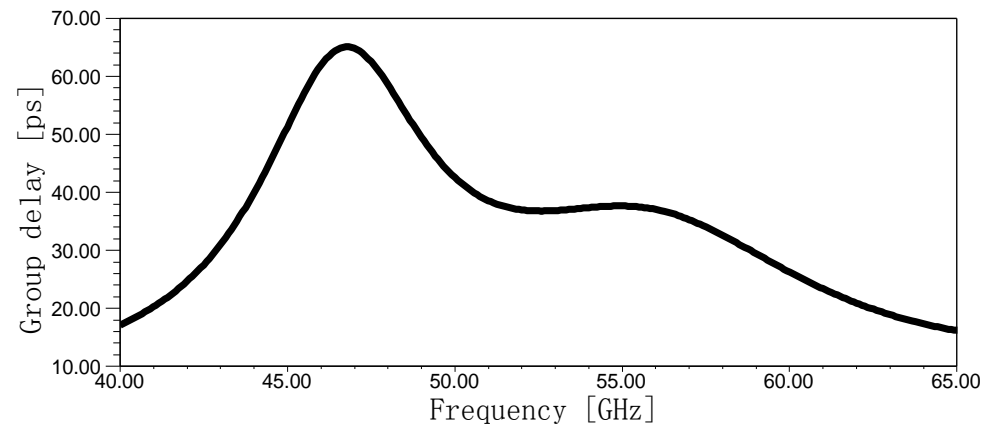

Figure 6. Group delay of the proposed band-pass filter. 
Science Foundation of Jiangxi Province of China (Grant Nos. 20122BAB201027) and Science and Technology Support Program of Jiangxi Province of China (Grant Nos. 20121BBE50028).

\section{References}

[1] Zhu, N.L., Feng, Q.Y. and Wu, Z. (2011) High-Gain Array Antenna Based on Composite Right/Left-Handed Transmission Line. Int. J. of Communications, Network and System Sciences, 4, 696-703. http://dx.doi.org/10.4236/ijcns.2011.411085

[2] Caloz, C. and Itoh, T. (2005) Electromagnetic Metamaterials: Transmission Line Theory and Microwave Applications. Wiley-IEEE Press, New York.

[3] Lai, A., Itoh, T. and Caloz, C. (2004) Composite Right/Left-Handed Transmission Line Metamaterials. IEEE Microwave Magazine, 5, 34-50. http://dx.doi.org/10.1109/MMW.2004.1337766

[4] Fei, W., Yu, H., Yeo, K.S., Liu, X. and Lim, W.M. (2012) A 44-to-60GHz, 9.7dBm P1dB, 7.1\% PAE Power Amplifier with 2D Distributed Power Combining by Metamaterial-Based Zero-Phase-Shifter in 65nm CMOS. Proceedings of IEEE MTT-S International Microwave Symposium Digest, Montreal, 17-22 June 2012.

[5] Moon, B.T. and Myung, N.H. (2014) Design of Low Phase-Noise Oscillator Based on a Hairpin-Shaped Resonator Using Composite Right/Left-Handed Transmission Line. IEEE Microwave and Wireless Components Letters, 24, 4446. http://dx.doi.org/10.1109/LMWC.2013.2287234

[6] Lee, C.J., Leong, K.M.K.H. and Itoh, T. (2005) Design of Resonant Small Antenna Using Composite Right/LeftHanded Transmission Line. Antennas and Propagation Society International Symposium, Washington, 218-221.

[7] Wang, Z.P., Han, Z.Y. and Guo, L.M. (2011) Miniaturized Zeroth-Order Resonator Based on Simplified CRLH TL Structure. Microwave. Optical. Technology Letters, 53, 848-852. http://dx.doi.org/10.1002/mop.25868

[8] García-García, J., Bonache, J. and Gil, I. (2006) Miniaturized Microstrip and CPW Filters Using Coupled Metamaterial Resonators. IEEE Transaction. Microwave Theory Technology, 54, 2628-2635. http://dx.doi.org/10.1109/TMTT.2006.872934

[9] Li, B., Dai, X.W., Wu, B. and Liang, C.H. (2007) Ultra Wideband Filter Design Based on Composite Right/Left Handed Transmission Line. Microwave and Optical Technology Letters, 49, 2379-2381. http://dx.doi.org/10.1002/mop.22733

[10] Han, W.J. and Feng, Y.J. (2008) Ultra-Wideband Bandpass Filter Using Simplified Left-Handed Transmission Line Structure. Microwave and Optical Technology Letters, 50, 2758-2762.

[11] Bahl, I.J. (2003) Lumped Elements for RF and Microwave Circuits. Artech House Publishers, Boston, London.

[12] Yin, J. and Luong, H.C. (2012) A 57.5-to-90.1 GHz Magnetically-Tuned Multimode CMOS VCO. IEEE, Custom Integrated Circuits Conference, USA, 9-12 September 2012, 1-4.

[13] Hu, X., Zhang, P. and He, S. (2006) Dual Structure of Composite Right/Left-Handed Transmission Line. Journal of Zhejiang University Science A, 7, 1777-1780. http://dx.doi.org/10.1631/jzus.2006.A1777 